\begin{tabular}{|c|c|}
\hline Title & $\begin{array}{l}\text { OsJA R1 and OsJA R2 are jasmonyl-L-isoleucine synthases involved in wound- and pathogen-induced jasmonic acid } \\
\text { signalling }\end{array}$ \\
\hline Author(s) & W akuta, Shinji; Suzuki, Erika; Saburi, W ataru; Matsuura, Hideyuki; Nabeta, Kensuke; Imai, Ryozo; Matsui, Hirokazu \\
\hline Citation & $\begin{array}{l}\text { Biochemical and Biophysical Research Communications, 409(4), } 634-639 \\
\text { https://doi.org/10.1016J.bbrc.2011.05.055 }\end{array}$ \\
\hline Issue Date & 2011-06-17 \\
\hline Doc URL & http:/hdl.handle.net/2115/46840 \\
\hline Tyре & article (author version) \\
\hline File Information & BBRC409-4_634-639.pdf \\
\hline
\end{tabular}

Instructions for use 


\section{OsJAR1 and OsJAR2 are jasmonyl-L-isoleucine synthases involved in wound- and pathogen-induced jasmonic acid signalling}

Shinji Wakuta ${ }^{\mathrm{a}}$, Erika Suzuki ${ }^{\mathrm{a}}$, Wataru Saburi ${ }^{\mathrm{a}, *}$, Hideyuki Matsuura ${ }^{\mathrm{a}}$, Kensuke Nabeta ${ }^{\mathrm{a}}$, Ryozo Imai ${ }^{\mathrm{b}}$ and Hirokazu Matsui ${ }^{\mathrm{a}}$

${ }^{a}$ Division of Applied Bioscience, Research Faculty of Agriculture, Hokkaido University, Sapporo 060-8589, Japan

${ }^{\mathrm{b}}$ National Agricultural Research Center of Hokkaido region, National Agriculture and Food Research Organization, Sapporo 062-8555, Japan

*Corresponding author. Tel./Fax: +81-11-706-2508

E-mail address: saburiw@chem.agr.hokudai.ac.jp

Division of Applied Bioscience, Research Faculty of Agriculture

Hokkaido University, N-9, W-9, Kita-Ku

Sapporo 060-8589, Japan

Key words: jasmonyl-L-isoleucine synthase; jasmonic acid; jasmonyl-L-isoleucine; wound response; response to blast infection

Abbreviations: IAA, indole-3-acetic acid; JA, jasmonic acid; JA-Ile, jasmonyl-L-isoleucine; MS/MS, tandem mass spectrometry; UPLC, ultra-performance liquid chromatography. 


\begin{abstract}
The synthesis of JA-Ile was catalysed by JA-Ile synthase, which is a member of the group I GH3 family of proteins. Here, we showed evidence that OsGH3.5 (OsJAR1) and OsGH3.3 (OsJAR2) are the functional JA-Ile synthases in rice, using recombinant proteins. The expression levels of OsJAR1 and OsJAR2 were induced in response to wounding with the concomitant accumulation of JA-Ile. In contrast, only the expression of OsJAR1 was associated with the accumulation of JA-Ile after blast infection. Our data suggest that these two JA-Ile synthases are differentially involved in the activation of JA signalling in response to wounding and pathogen challenge in rice.
\end{abstract}


Highlights

1) Two genes from rice plant, OsGH3.5 (OsJAR1) and OsGH3.3 (OsJAR2), had been confirmed to encode functional JA-Ile synthase.

2) The expression of OsJAR1 and OsJAR2 were induced in response to wounding with the concomitant accumulation of JA-Ile.

3) Only the expression of OsJAR1 was associated with the accumulation of JA-Ile after blast infection. 


\section{OsJAR1 and OsJAR2 are jasmonyl-L-isoleucine synthases involved in wound- and pathogen-induced jasmonic acid signalling}

Shinji Wakuta ${ }^{a}$, Erika Suzuki $^{a}$, Wataru Saburi ${ }^{\mathrm{a}, *}$, Hideyuki Matsuura $^{\mathrm{a}}$, Kensuke Nabeta $^{\mathrm{a}}$, Ryozo Imai ${ }^{\mathrm{b}}$ and Hirokazu Matsui ${ }^{\mathrm{a}}$

${ }^{a}$ Division of Applied Bioscience, Research Faculty of Agriculture, Hokkaido University, Sapporo 060-8589, Japan

${ }^{\mathrm{b}}$ National Agricultural Research Center of Hokkaido region, National Agriculture and Food Research Organization, Sapporo 062-8555, Japan

*Corresponding author. Tel./Fax: +81-11-706-2508

E-mail address: saburiw@chem.agr.hokudai.ac.jp

Division of Applied Bioscience, Research Faculty of Agriculture

Hokkaido University, N-9, W-9, Kita-Ku

Sapporo 060-8589, Japan

Key words: jasmonyl-L-isoleucine synthase; jasmonic acid; jasmonyl-L-isoleucine; wound response; response to blast infection

Abbreviations: IAA, indole-3-acetic acid; JA, jasmonic acid; JA-Ile, jasmonyl-L-isoleucine; MS/MS, tandem mass spectrometry; UPLC, ultra-performance liquid chromatography. 


\begin{abstract}
The synthesis of JA-Ile was catalysed by JA-Ile synthase, which is a member of the group I GH3 family of proteins. Here, we showed evidence that OsGH3.5 (OsJAR1) and OsGH3.3 (OsJAR2) are the functional JA-Ile synthases in rice, using recombinant proteins. The expression levels of OsJAR1 and OsJAR2 were induced in response to wounding with the concomitant accumulation of JA-Ile. In contrast, only the expression of OsJAR1 was associated with the accumulation of JA-Ile after blast infection. Our data suggest that these two JA-Ile synthases are differentially involved in the activation of JA signalling in response to wounding and pathogen challenge in rice.
\end{abstract}




\section{Introduction}

Jasmonic acid (JA) is an oxylipin phytohormone that is essential for regulating stress responses against insect herbivory and pathogenic attack in plants [1,2]. JA also regulates plant growth and development, including root elongation, fruit ripening, and pollen maturation [3-6]. JA undergoes various modifications that are implicated in activation, inactivation, storage, transport, and volatilisation. Among these modifications, amino acid conjugation controls the physiological activity of JA. JA in conjugation with Ile, Leu, Val, Phe, and ACC have been detected in a wide range of plant species [7,8]. Among them, JA-Ile is a major active compound in JA signalling $[9,10]$.

Similar to auxin, the amino acid conjugation of JA is catalysed by GH3 proteins. The GH3 protein was first isolated from Glycine max as an early auxin-responsive gene [11]. GH3 is encoded by a multigene family in various plant species, and the protein sequences are classified into three groups [12,13]. One of the group I GH3 proteins in Arabidopsis, AtJAR1, has been shown to synthesise JA-amino acid conjugates, such as JA-Ile, JA-Leu, and JA-Val [14], while group II proteins produce indole-3-acetic acid (IAA) conjugates, such as IAA-Asp, IAA-Glu, and IAA-Ala [15]. Group III proteins are involved in the conjugation of amino acids to 4-substituted benzoate [16].

The physiological roles of group I GH3 proteins have been elucidated in the Arabidopsis jar1 mutant, which shows decreased sensitivity to exogenous JA [3,17] and decreased levels of JA-Ile [14]. JAR1 is involved in resistance to the soil fungus Pythium irregular [18], induced systemic resistance to rhizobacteria [19,20], and protection against ozone damage [21,22]. Group I GH3 proteins with JA-Ile synthase activity have been identified in Nicotiana attenuate (JAR4 and JAR6) and tomato (S1JAR1), and they are involved in the activation of defence systems after wounding [23-25].

Three group I GH3 proteins, GH3.3, GH3.5, and GH3.12, are encoded by the rice genome. Although GH3.5 (OsJAR1) has been implicated in phytochrome and jasmonic acid signalling [26], no active JA-Ile synthase has been identified thus far in rice. In this study, we report that OsJAR1 and OsJAR2 (GH3.3) are the functional JA-Ile synthases, and their expression correlates with JA-Ile accumulation after wounding and pathogen 
attack.

\section{Materials and methods}

\subsection{Plant materials, growth conditions, and stress treatments}

Seeds of $O$. sativa L. cv. Nipponbare were surface-sterilised in $70 \%$ ethanol for 3 min and further sterilised in 50\% commercial bleach for $30 \mathrm{~min}$. The sterilised seeds were then soaked in distilled water for 4 days and set to germinate in the dark at $20^{\circ} \mathrm{C}$. Germinated seeds were uniformly distributed into a seed bed. Seeds were then grown under a $10.5 \mathrm{~h}$ light period in a growth chamber at $25^{\circ} \mathrm{C}, 60 \%$ humidity, and $530 \mu \mathrm{mol} / \mathrm{m}^{2} / \mathrm{s}$ illumination.

At the fourth leaf stage, seedlings were subjected to wounding or pathogenic stress treatments. Seedlings were nipped tightly with a pair of tweezers. Shoots of the treated seedlings were collected at 0 (control), $0.5,1,2$, and $4 \mathrm{~h}$ after the wounding treatment, immediately frozen in liquid nitrogen, and stored at $-80^{\circ} \mathrm{C}$ until further analysis.

For the blast infection, Magnaporthe grisea (race, 007) was grown on an oatmeal medium (Difco Laboratories, Detroit, MI, USA) for 1 week at $25^{\circ} \mathrm{C}$ in the dark, and then spore formation was induced under a $15 \mathrm{~W}$ BLB light (FL15S, BLB15W; Toshiba, Tokyo, Japan) for 4 days at $25^{\circ} \mathrm{C}$. A spore suspension $\left(2.5 \times 10^{7}\right.$ spores $\left./ \mathrm{ml}\right)$ in $0.05 \%$ Tween-20 was sprayed on the seedlings at the fifth leaf stage. The sprayed plants were incubated at $25^{\circ} \mathrm{C}$ with high humidity in the dark for $20 \mathrm{~h}$ and then moved back to the original conditions. Shoots of the treated seedlings were collected at 0 (control), 24, 48, and $72 \mathrm{~h}$ after the blast treatment and stored as mentioned above.

\section{2. cDNA cloning of three $c D N A$ s from the group I GH3}

Based on the amino acid sequences of OsJAR1, OsJAR2, and OsJAR3 obtained from the MSU-RGAP database [27], primers were designed and are shown in Supplementary Table 1. Total RNA was extracted from the rice leaf sheath and roots by RNAqueous (Applied Biosystems, Foster City, CA), and cDNA was synthesised by reverse transcription, using the BcaBest RNA PCR Kit Ver. 1.1(Takara Bio, Kyoto, Japan). Prime Star Polymerase 
(Takara Bio) was used for the cDNA amplification. The reaction temperatures were set to $98^{\circ} \mathrm{C}$ for $10 \mathrm{~s}$ and $68^{\circ} \mathrm{C}$ for $1 \mathrm{~min} 30 \mathrm{~s}$ with 30 cycles. The DNA fragments were inserted into a pBluescript II SK (+) plasmid (Stratagene, La Jolla, CA, USA), and sequenced to verify that no errors had occurred.

\subsection{Construction of expression vectors}

Expression plasmids were constructed to produce recombinant OsJAR1 and OsJAR2 as GST and 6x His tagged proteins. The ORFs of OsJAR1 and OsJAR2 were amplified with PciI and HindIII linker sequences from the cDNA clones, respectively. These PCR products were inserted into pET42a (Novagen, Madison, WI, USA) via NcoI and HindIII.

\subsection{Induction of expression of OsJAR1 and OsJAR2 and analysis of JA-Ile formation in} recombinant E. coli cells

E. coli cells carrying each expression vector were cultured overnight in $2 \mathrm{ml}$ of Luria-Bertani (LB) medium, containing $50 \mu \mathrm{g} / \mathrm{ml}$ of kanamycin at $37^{\circ} \mathrm{C}$. The culture was transferred into fresh LB medium $(2 \mathrm{ml})$ and incubated until the absorbance at $600 \mathrm{~nm}$ reached 0.1 . Then, isopropyl thio- $\beta$-D-galactoside was added at a final concentration of 1 $\mathrm{mM}$, and the culture was incubated at $37^{\circ} \mathrm{C}$ for $2 \mathrm{~h}$ to induce protein production. Subsequently, JA was added to the culture at a final concentration of $0.1 \mathrm{mM}$ and incubation was conducted at $37^{\circ} \mathrm{C}$ for $3 \mathrm{~h}$ with shaking. After extraction with an equal volume of $\mathrm{MeOH}$ and centrifugation at $6,000 \mathrm{x} g$ for $10 \mathrm{~min}$, the supernatant was collected. As an internal standard, JA-Ile $d-6$ ( $5 \mathrm{ng}$ ) was added to the extract, and the mixture was analysed, using the ultra-performance liquid chromatography tandem mass spectrometry (UPLC-MS/MS) system. The UPLC-MS/MS instrumentation conditions for the analysis and synthesis of JA and JA-amino acid conjugates were set as previously described $[28,29]$.

\subsection{Analyses of the endogenous JA and JA-amino acid conjugates after wounding}

The frozen sample was crushed and soaked in $15 \mathrm{ml}$ of $80 \%$ aqueous $\mathrm{MeOH}$ with $0.5 \%$ acetic acid for $12 \mathrm{~h}$. The mixture was filtered to give a crude extract, and JA $d-6$ (400 ng), 
JA-Ile $d$-6 (100 ng), JA-Leu d-6 (100 ng), JA-Trp d-6 (100 ng), JA-Gly d-6 (100 ng), JA-Val $d-6$ (100 ng), JA-Phe $d-6$ (100 ng), and JA-ACC $d-6$ (100 ng) were added to the extract as internal standards, and analysed as previously described[28,29].

\subsection{Real-time PCR analysis}

Total RNA from shoots treated with wounding and blast infection were prepared with RNAqueous as described above, and cDNAs were synthesised from the RNA, using random hexamer primers and Superscript III (Invitrogen, Carlsbad, CA, USA). The real-time PCR reaction was performed, using SYBR Premix Ex Taq (Takara Bio), and the signals were detected on a Smart Cycler II System (Takara Bio). The sequences of each specific primer are described in Supplementary Table 2. The real-time PCR reaction was normalised, using the rice $18 S$ rRNA gene as an internal control. The results shown are the mean values of at least three independent RNA samples.

\section{Results}

\subsection{Phylogenetic relationship of the group I GH3 proteins in plants}

To identify the sequence diversity of group I GH3 proteins in plant genomes, sequences were collected from public genome databases for monocots (Oryza sativa, Zea mays, Brachypodium distachyon, and Sorghum bicolor) and dicots (Arabidopsis thaliana, Vitis vinifera, Glycine max, and Populus trichocarpa). In total, 35 protein sequences were selected for the multiple alignment and phylogenetic analyses, using the Clustal W software [30]. The phylogenetic tree identified four subgroups within the group I GH3 proteins (Fig. 1). Five enzymatically active JA-Ile synthases that had been previously characterised fell into the dicot subgroup I. The three group I GH3 proteins from rice, OsJAR1/OsGH3.5, OsJAR2/OsGH3.3, and OsJAR3/OsGH3.12 were classified into different subgroups. OsJAR1 was classified into the monocot subgroup I, while OsJAR2 and OsJAR3 were classified into the monocot subgroup II. 


\subsection{Isolation of three cDNAs encoding OsJAR1, OsJAR2, and OsJAR3}

To identify the functional JA-Ile synthase genes in rice, the three OsJAR cDNAs were isolated. RT-PCR was performed with specific primer sets that were designed using genome annotation information available at MSU-RGAP (Release 6.1). Because two mRNA variants were predicted for OsJAR2 by the MSU-RGAP database, both OsJAR2.1 and OsJAR2.2 cDNAs were isolated. The OsJAR3 cDNA could not be amplified from the leaf sheath. Among the several tissues tested, the OsJAR3 cDNA could only be amplified from a callus containing two introns. Therefore, we assumed that OsJAR3 is not functional in most tissues. Both OsJAR1 and OsJAR2.1 have three motifs involved in ATP/AMP binding [31] (Supplementary Fig. 1): motif 1, SSGTT(H/Q)QKRK; motif 2, YGASEGW; and motif 3, YRLGDVVK. However, OsJAR2.2 does not contain motif 3, suggesting the possibility that OsJAR2.2 may lack enzymatic activity. The amino acid sequences of OsJAR1 and OsJAR2.1 share $60 \%$ and 59\% identity to AtJAR1, respectively.

\subsection{Enzymatic production of JA-Ile by OsJAR1 and OsJAR2.1}

To examine the JA-Ile-producing activities of OsJAR1, OsJAR2.1, and OsJAR2.2, E. coli cells expressing these genes were incubated with JA, and the production of JA-Ile was determined by UPLC-MS/MS (Fig. 2). The analysis of the reaction products in the culture supernatant clearly demonstrated that OsJAR1 and OsJAR2.1 catalyse the conjugation of JA to Ile, using endogenous ATP and Ile from E. coli. In contrast, no JA-Ile production was detected in the cells producing OsJAR2.2 (data not shown). Therefore, we concluded that OsJAR2.1 is a functional OsJAR2 transcript.

\subsection{Induction of mRNAs encoding OsJAR1 and OsJAR2 by wounding}

The up-regulation of JA-Ile synthase mRNA and accumulation of JA-Ile were concomitantly observed, following leaf wounding in Arabidopsis and tomato [25,29]. Therefore, the expression levels of OsJAR1 and OsJAR2 and accumulation of JA-Ile in response to wounding stress were assessed. A real-time PCR analysis showed that the transcripts of OsJAR1 and OsJAR2 were induced transiently, reaching peaks at $1 \mathrm{~h}$ and 0.5 
h, respectively (Fig. 3A). Analyses of endogenous JA, JA-Ile, and other JA-conjugates in wounded rice plants showed that the endogenous levels of JA and JA-Ile were increased after wounding. Maximum levels of accumulation of JA (3.29 nmol/g FW) and JA-Ile $(1.59 \mathrm{nmol} / \mathrm{g} \mathrm{FW})$ were observed at $1 \mathrm{~h}$ after wounding (Fig. 3B). Among the analysed JA-conjugates, JA-Val $(0.12 \mathrm{nmol} / \mathrm{g} \mathrm{FW})$ and JA-Phe $(<0.01 \mathrm{nmol} / \mathrm{g} \mathrm{FW})$ were detected, but they were induced at much lower levels than JA-Ile (Fig. 3C). These data indicate that OsJAR1 and OsJAR2 are both involved in the synthesis of JA-Ile in response to wounding stress.

\subsection{Determination of mRNA levels of OsJAR1 and OsJAR2 after blast infection}

To elucidate the influence of pathogenic attack on jasmonic acid signalling, blast infections were performed by spraying intact plants with a spore suspension of rice blasts. The real-time PCR analysis revealed that OsJAR1 mRNA levels increased gradually from 48 to $72 \mathrm{~h}$ after the blast spray, while OsJAR2 expression was not significantly altered (Fig. 4A). Endogenous JA-Ile levels were also induced from 48 to $72 \mathrm{~h}$ after infection and reached maximum levels $(0.5 \mathrm{nmol} / \mathrm{g} \mathrm{FW})$ at $72 \mathrm{~h}$ (Fig. 4B). In infected leaves, 0.069 nmol/g FW of JA-Val was also detected at 72 h (Fig. 4C), while JA-Phe was not detected under these conditions. These data showed that OsJAR1 mainly produces JA-Ile in response to pathogen attack. JA levels gradually increased from 0 to $72 \mathrm{~h}$ after inoculation, while a dramatic increase in JA-Ile was observed after $24 \mathrm{~h}$ (Fig. 4).

\section{Discussion}

OsJAR1 (OsGH3.5) has been implicated in phytochrome and JA signalling [26]. However, enzymes that synthesise JA-amino acid conjugates have not been identified in rice. In this study, we demonstrated that OsJAR1 (OsGH3.5) and OsJAR2 (OsGH3.3), belonging to the group I GH3 family proteins, exert their activities to form JA-Ile from JA and Ile in response to wounding and pathogenic stress in rice.

A phylogenetic analysis of group I GH3 proteins from eight plant species whose genome sequences are known revealed that they are divided into four subgroups. Five previously 
characterised group I GH3s, AtJAR1, SIJAR1, NgJAR1, NaJAR4, and NaJAR6, are all clustered into the dicot subgroup I (Fig. 2). It was not known if the other subgroup members within the group I GH3 proteins also displayed JA-Ile synthase activities. Because OsJAR1 and OsJAR2 are positioned in distinct monocot subgroups (Fig. 1), our data suggest the possibility that a wider cluster within the group I GH3 proteins displays JA-Ile synthase activity.

In contrast to OsJAR1 and OsJAR2, the existence of the OsJAR3 transcript was questionable. No corresponding full-length cDNA or EST for OsJAR3 could be identified in the databases [13]. The OsJAR3 mRNA was not detectable in tissues, including those from the shoot and roots, where OsJAR1 and OsJAR2 mRNA are present [13]. However, another group reported the detection of OsJAR3 mRNA in the roots [12]. In this study, RT-PCR was performed, using mRNA from the roots, shoot, leaf sheath, leaf blade, panicles, immature seeds and callus cells, but only the unspliced form of OsJAR3 transcripts were detected in the callus. The resulting transcript encodes a truncated protein due to a nonsense mutation. The putative spliced form of the OsJAR3 mRNA, encoding a protein with three conserved ATP/AMP binding motifs, may exist in tissues under specific stress conditions or in certain developmental stages. However, it is also possible that OsJAR3 is a pseudogene because there is one group I GH3 protein in each subgroup in Brachypodium.

The expressions of OsJAR1 and OsJAR2 were both rapidly and transiently induced in response to wounding (Fig. 3). The accumulation pattern of JA-Ile in rice leaves after wounding was in accordance with the expression patterns of OsJAR1 and OsJAR2 (Fig. 3). Similarly, the accumulation of JA-Ile in response to wounding stress is rapid and transient in Arabidopsis, tobacco, and tomato leaves [23,25,32]. These observations suggest that the transcriptional activation of OsJAR1 and OsJAR2 is required for the production of JA-Ile, which turns on jasmonic acid signalling in rice as was reported for Arabidopsis. The formation of JA-Leu, JA-Trp, JA-Gly, JA-Val, JA-Phe, and JA-ACC were monitored after wounding, and JA-Val and JA-Phe could be detected at low levels compared to JA-Ile.

The accumulation of JA-Ile was also observed in blast-infected leaves. However, the 
accumulation pattern was quite different from that caused by wounding. Compared with wound stress, JA-Ile accumulated slowly and continuously and reached higher levels than JA. Relatively slow accumulations of JA and JA-Ile may be due to the fact that pathogen infection occurs progressively at the site of inoculation. A dramatic increase in JA-Ile levels was observed after $24 \mathrm{~h}$ (Fig. 4). The correlation of OsJAR1 expression and JA-Ile accumulation suggests that OsJAR1 is involved in JA-Ile synthesis during blast infection.

In summary, we have demonstrated that two functionally active JA-Ile synthases are present in rice. They are differentially regulated in response to multiple stressors. Further enzymatic and physiological characterisations of OsJAR1 and OsJAR2 are required to understand the role of JA signalling in rice.

\section{References}

[1] M. McConn, R.A. Creelman, E. Bell, J.E. Mullet, J. Browse, Jasmonate is essential for insect defense in Arabidopsis, Proc. Natl. Acad. Sci. USA 94 (1997) 5473-5477.

[2] P. Vijayan, J. Shockey, C.A. Lévesque, R.J. Cook, J. Browse, A role for jasmonate in pathogen defense of Arabidopsis, Proc. Natl. Acad. Sci. USA 95 (1998) 7209-7214.

[3] P.E. Staswick, W. Su, S.H. Howell, Methyl jasmonate inhibition of root growth and induction of a leaf protein are decreased in an Arabidopsis thaliana mutant, Proc. Natl. Acad. Sci. USA 89 (1992) 6837-6840.

[4] X. Fan, J.P. Mattheis, J.K. Fellman, A role for jasmonates in climacteric fruit ripening, Planta 204 (1998) 444-449.

[5] A. Stintzi, J. Browse, The Arabidopsis male-sterile mutant, opr3, lacks the 12-oxophytodienoic acid reductase required for jasmonate synthesis, Proc. Natl. Acad. Sci. USA 97 (2000) 10625-10630.

[6] C. Wasternack, Jasmonates: An update on biosynthesis, signal transduction and action in plant stress response, growth and development, Annals of Botany 100 (2007) 681-697. [7] R. Kramell, R. Atzorn, G. Schneider, O. Miersch, C. Bruckner, G. Schmidt, B. Parthier, Occurrence and identification of jasmonic acid and its amino acid conjugates induced by osmotic stress in barley leaf tissue, J. Plant Growth Regul. 14 , (1995) 29-36. 
[8] B. Hause, W. Maier, O. Miersch, R. Kramell, D. Strack, Induction of jasmonate biosynthesis in arbuscular mycorrhizal barley roots, Plant Physiol. 130 (2002) 1213-1220. [9] A. Chini, S. Fonseca, G. Fernandez, B. Adie, J.M. Chico, O. Lorenzo, G. Gracia-Casado, I. Lopez-Vidriero, F.M. Lozano, M.R. Ponce, J.L. Micol, R. Solano, The JAZ family of repressors is the missing link in jasmonate signalling, Nature 448 (2007) 666-671.

[10] B. Thines, L. Katsir, M. Melotto, Y. Niu, A. Mandaokar, G. Liu, K. Nomura, S. Y. He, G.A. Howe, J. Browse, JAZ repressor proteins are targets of the SCFCOI1 complex during jasmonate signalling, Nature 448 (2007) 661-665.

[11] G. Hagen, T.J. Guilfoyle, Rapid induction of selective transcription by auxins, Mol. Cell Biol. 5 (1985) 1197-1203.

[12] M. Jain, N. Kaur, A.K. Tyagi, J.P. Khurana, J.P. The auxin-responsive GH3 gene family in rice (Oryza sativa), Funct. Integr Genomics 6 (2006) 36-46.

[13] J. Terol, C. Domingo, M. Talon, The GH3 family in plants: Genome wide analysis in rice and evolutionary history based on EST analysis, Gene 371 (2006) 279-290.

[14] P.E. Staswick, I. Tiryaki, The oxylipin signal jasmonic acid is activated by an enzyme that conjugates it to isoleucine in Arabidopsis, Plant Cell 16 (2004) 2117-2127.

[15] P.E. Staswick, B. Serban, M. Rowe, I. Tiryaki, M.T. Maldonado, M.C. Maldonado, W.P. Suza, Characterization of an Arabidopsis enzyme family that conjugates amino acids to indole-3-acetic acid, Plant Cell 17 (2005) 616-627.

[16] R.A. Okrent, M.D. Brooks, M.C. Wildermuth, Arabidopsis GH3.12 (PBS3) conjugates amino acids to 4 -substituted benzoates and is inhibited by salicylate, J. Biol. Chem. 284 (2009) 9742-9754.

[17] P.E. Staswick, I. Tiryaki, M. Rowe, M. Jasmonate response locus JAR1 and several related Arabidopsis genes encode enzymes of the firefly luciferase superfamily that show activity on jasmonic, salicylic, and indole-3-acetic acids in an assay for adenylation, Plant Cell 14 (2002) 1405-1415.

[18] P.E. Staswick, G.Y. Yuen, C.C. Lehman, Jasmonate signaling mutants of Arabidopsis are susceptible to the soil fungus Pyhium irregular, Plant J. 15 (1998) 747-754. 
[19] C.M.J. Pieterse, S.C.M. van Wees, J.A. van Pelt, M. Knoester, R. Laan, H. Gerrits, P.J. Weisbeek, L.C. van Loon, A novel signaling pathway controlling induced systemic resistance in Arabidopsis, Plant Cell 10 (1998) 1571-1580.

[20] J.D. Clarke, S.M. Volko, H. Ledford, F.M. Ausubel, X. Dong, Roles of salicylic acid, jasmonic acid, and ethylene in cpr-induced resistance in Arabidopsis, Plant Cell 12 (2000) 2175-2190.

[21] K. Overmyer, H. Tuominen, R. Kettunen, C. Betz, C. Langebartels, H. Sandermann, J. Kangasjarvi, Ozone-sensitive Arabidopsis rcd1 mutant reveals opposite roles for ethylene and jasmonate signaling pathways in regulating superoxide-dependent cell death, Plant Cell 12 (2000) 1849-1862.

[22] M.V. Rao, H. Lee, R.A. Creelman, J.E. Mullet, K.R. Davis, Jasmonic acid signaling modulates ozone-induced hypersensitive cell death, Plant Cell 12 (2000) 1633-1646. [23] J.H. Kang, L. Wang, A. Giri, I.T. Baldwin, Silencing threonine deaminase and JAR4 in Nicotiana attenuata impairs jasmonic acid-isoleucine-mediated defences against Manduca sexta, Plant Cell 18 (2006) 3303-3320.

[24] L. Wang, S. Allmann, J. Wu, I.T. Baldwin, Comparisons of LIPOXYGENASE3- and JASMONATE-RESISTANT4/6-silenced plants reveal that jasmonic acid and jasmonic acid-amino acid conjugates play different roles in herbivore resistance of Nicotiana attenuate, Plant Physiol. 146 (2007) 904-915.

[25] W.P. Suza, M.L. Rowe, M. Hamberg, P.E. Staswick, A tomato enzyme synthesizes (+)-7-iso-jasmonoyl-L-isoleucine in wounded leaves, Planta 231 (2010) 717-728.

[26] M. Riemann, M. Riemann, M. Takano, Rice JASMONATE RESISTANT 1 is involved in phytochrome and jasmonate signalling, Plant Cell Environ. 31 (2008) 783-792. [27] S. Ouyang, W. Zhu, J. Hamilton, H. Lin, M. Campbell, K. Childs, T. Thibaud-Nissen, R.L. Malek, Y. Lee, L. Zheng, J. Orvis, B. Haas, J. Wortman, C.R. Buell, The TIGR rice genome annotation resource: improvements and new features, Nucleic Acids Res. 35 (2007) 883-887.

[28] H. Matsuura, A. Aoi, C. Satou, M. Nakaya, C. Masuta, K. Nabeta. Simultaneous UPLC MS/MS analysis of endogenous jasmonic acid, salicylic acid, and their related 
compounds, Plant Growth Regul. 57 (2009) 293-301.

[29] C. Sato, Y. Seto, K. Nabeta, H. Matsuura, Kinetics of the accumulation of jasmonic acid and its derivatives in systemic leaves of tobacco (Nicotiana tabacum cv. Xanthi nc) and translocation of derterium-labeled jasmonic acid from the wounding site to the systemic site, Biosci. Biotechnol. Biochem. 73 (2009) 1962-1970.

[30] J.D. Thompson, D.G. Higgins, T.J. Gibson, CLUSTAL W: improving the sensitivity of progressive multiple sequence alignment through sequence weighting, position-specific gap penalties and weight matrix choice, Nucleic Acids Res. 22 (1994) 4673-4680.

[31] K.H. Chang, H. Xiang, D. Dunaway, Mariano, Acyl-adenylate motif of the acyl-adenylate/thioester-forming enzyme superfamily: a site-directed mutagenesis study with the Pseudomonas sp. strain CBS3 4-chlorobenzoate: coenzyme A ligase, Biochemistry 36 (1997) 15650-15659.

[32] W.P. Suza, P.E. Staswick, The role of JAR1 in jasmonoyl-L-isoleucine production during Arabidopsis wound response, Planta 227 (2008) 1221-1232.

[33] N. Saitou, M. Nei, The neighbor-joining method: a new method for reconstructing phylogenetic trees, Mol. Biol. Evol. 4 (1987) 406-425.

[34] Arabidopsis Genome Initiative, Analysis of the genome sequence of the flowering plant Arabidopsis thaliana, Nature 408 (2000) 796-815.

[35] G.A. Tuskan, et al. The genome of black cottonwood, Populus trichocarpa (Torr. \& Gray), Science 313 (2006) 1596-1604.

[36] Grapevine Genome Characterization, The grapevine genome sequence suggests ancestral hexaploidization in major angiosperm phyla, Nature 449 (2007) 463-467.

[37] A.H. Paterson, et al, The Sorghum bicolor genome and the diversification of grasses, Nature 457 (2009) 551-556.

[38] P.S. Schnable, et al, The B73 maize genome: complexity, diversity, and dynamics, Science 326 (2009) 1112-1115.

[39] J. Schmutz, et al, Genome sequence of the palaeopolyploid soybean, Nature 463 (2010) 178-183.

[40] International Brachypodium Initiative, Genome sequencing and analysis of the model 
grass Brachypodium distachyon, Nature 463 (2010) 763-768.

[41] M. Jain, A. Nijhawan, A.K. Tyagi, J.P. Khurana, Validation of housekeeping genes as internal control for studying gene expression in rice by quantitative real-time PCR, Biochem. Biophys. Res. Commun. 345 (2006) 646-651.

\section{Figure captions}

Fig. 1. A phylogenetic tree of the amino acid sequences from the group I GH3 family proteins from various plant species.

The tree was constructed, using ClustalX (version 2.0.11) (neighbour-joining method; [33]), and visualised with $\mathrm{NJ}$ plot, using amino acid sequences gathered from various databases [32,34-40]. Numbers indicate bootstrap values. The positions of proteins targeted in this study are indicated with open triangles: OsJAR1 (LOC_Os05g50890), OsJAR2 (LOC_Os01g12160), and OsJAR3 (LOC_Os11g08340). Known JA-Ile synthase proteins are indicated by closed triangles: AtJAR1 (Arabidopsis thaliana); NaJAR4 and NaJAR6 (Nicotiana attenuata); NgJAR1 (Nicotiana glutinosa); and SlJAR1 (Solanum lycopersicum). Protein sequences were obtained from genome databases of Glycine max (Glyma16g03010, Glyma07g06370, Glyma19g44310, Glyma03g41700, Glyma13g37550, Glyma12g32910, Glyma12g11890, and Glyma06g45120), Populus trichocarpa (POPTR_0002s16960, POPTR_0014s09120, POPTR_13s14050, and POPTR_0019s13450), Visis vinifera (GSVIVT01027057001 and GSVIVT01030558001), Brachypodium distachyon (Bradi2g14830 and Bradi2g07230), Zea mays (GRMZM2G091276_P02, GRMZM2G162413_P01, GRMZM2G001421_P01, GRMZM2G060991_P01, and GRMZM2G061005_P01), Sorghum bicolor (Sb03g028240, Sb09g030050, Sb03g001490, and Sb03g001500), and Arabidopsis thaliana (AtGH3.10: At4g03400).

Fig. 2. Metabolism of JA in E. coli cells carrying the OsJAR1 and OsJAR2 genes.

The trace chromatogram in the selected reaction monitoring mode shows the ion peaks $\mathrm{m} / \mathrm{z}$ 328.03/129.68 for JA-Ile $d-6$ and $\mathrm{m} / \mathrm{z}$ 322.03/129.68 for JA-Ile. JA-Ile $d-6$ was used 
as an internal standard. The metabolism in culture supernatant of $E$. coli cells with pET42a as a control (A), pET42a:OsJAR1 (B), and pET42a:OsJAR2 (C) were analysed.

Fig. 3. The expression levels of OsJAR1 and OsJAR2 mRNA and the accumulation of JA and JA-amino acid conjugates in response to wounding.

(A) The accumulation of OsJAR1 and OsJAR2 transcripts after mechanical wounding was quantified by quantitative real-time RT-PCR with the specific primer sets. Data were normalised with respect to $18 \mathrm{~s} r R N A$ levels [41]. The relative amount of each mRNA was calculated, using intact (unwounded) plants as standards. Each value is the mean \pm S.D. of three independent experiments. (B),(C) Endogenous amounts of JA (triangles), JA-Ile (circles), and JA-Val (squares) were assayed in plants after wound treatments by the UPLC-MS/MS system. JA-Val was not detected before wounding. Data are mean \pm S.D. for three independent experiments.

Fig. 4. The expression levels of OsJAR1 and OsJAR2 mRNA and the accumulation of JA and JA-amino acid conjugates in response to blast infection.

(A) The accumulation of OsJAR1 and OsJAR2 transcripts after blast infection was quantified by quantitative real-time RT-PCR with the specific primer sets. Data were normalised with respect to $18 \mathrm{~s} r R N A$ levels [41]. The relative amount of each mRNA was calculated against the amount in intact (uninfected) rice. Each value is the mean \pm S.D. of three independent experiments. (B), (C) Endogenous levels of JA (triangles), JA-Ile (circles), and JA-Val (squares) were assayed in plants after infection, using the UPLC-MS/MS system. Data are the mean \pm S.D. of three independent experiments. 
AtGH3.2

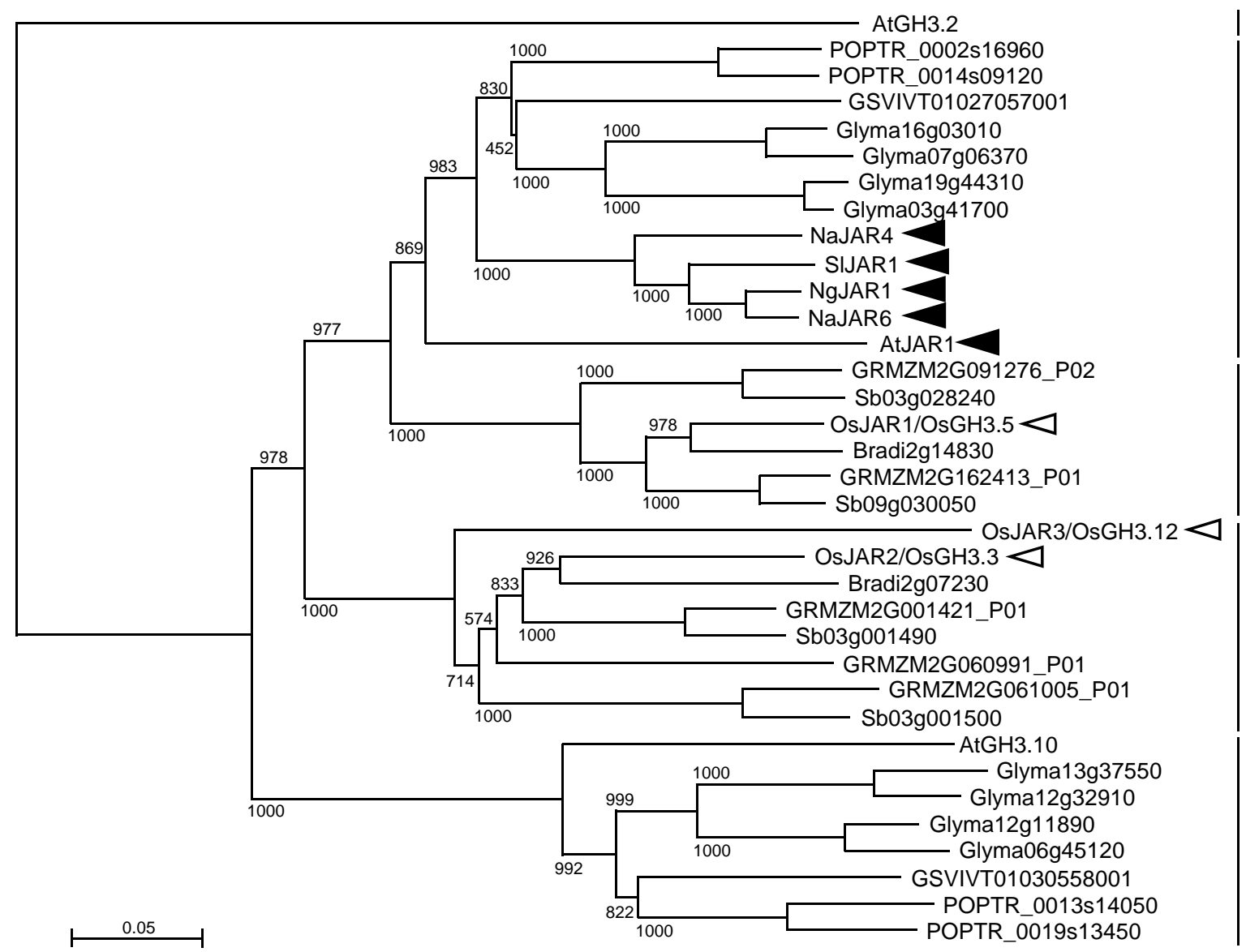

GH3 family group II

GH3 family group I dicot subgroup I

GH3 family group I monocot subgroup I

GH3 family group I monocot subgroup II

GH3 family group I dicot subgroup II

Fig. 1. 

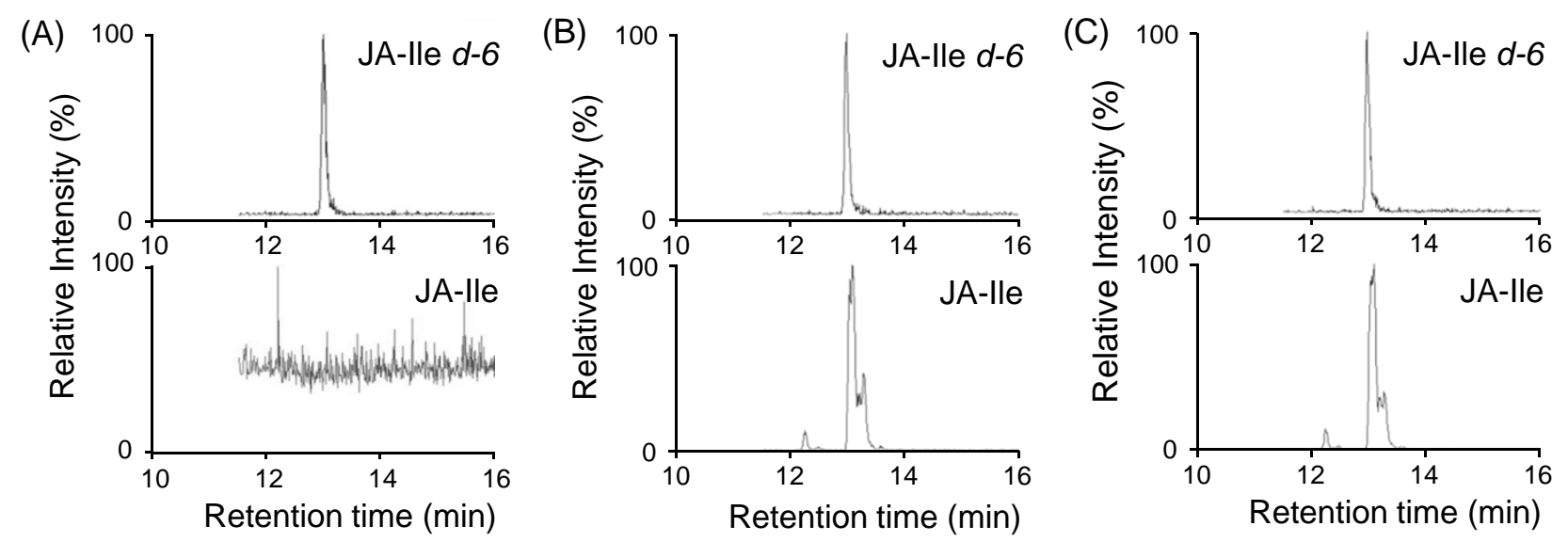

Fig. 2. 

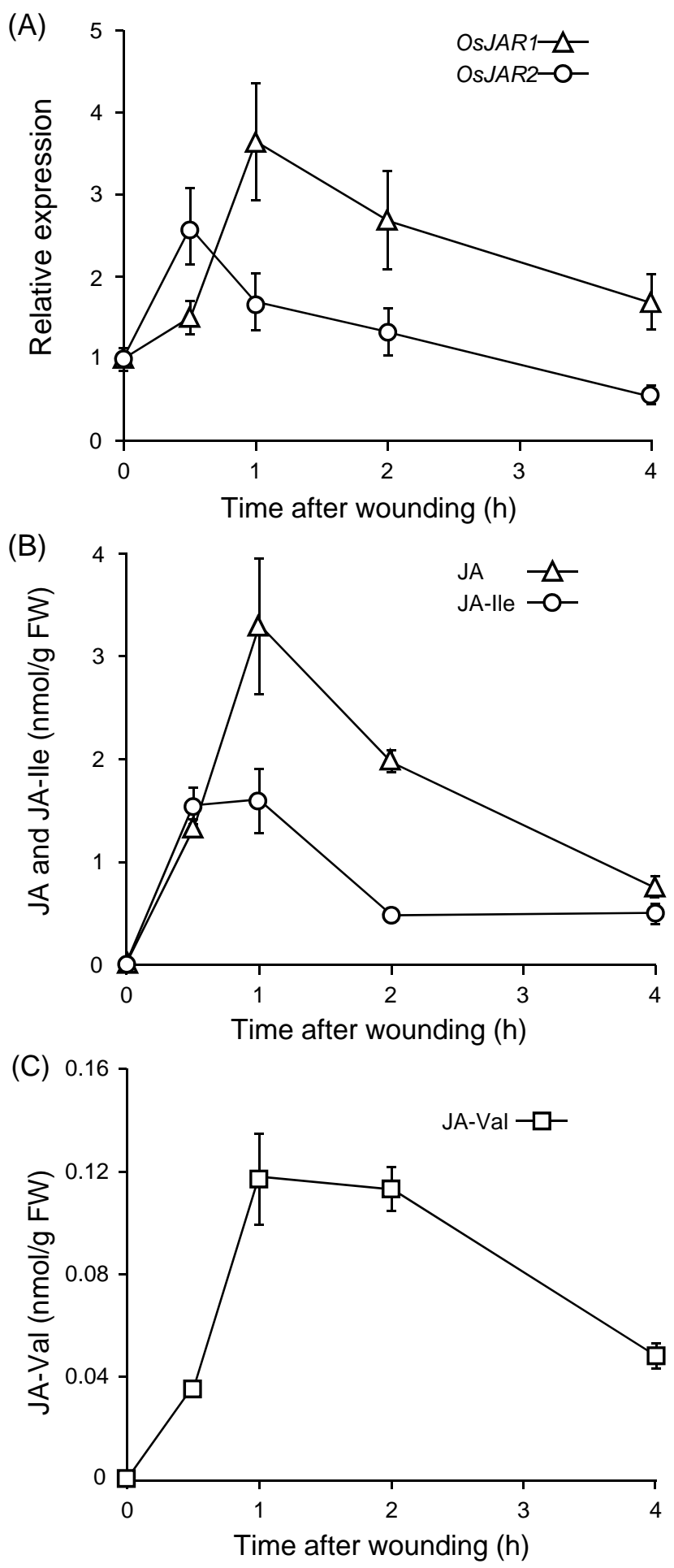

Fig. 3. 

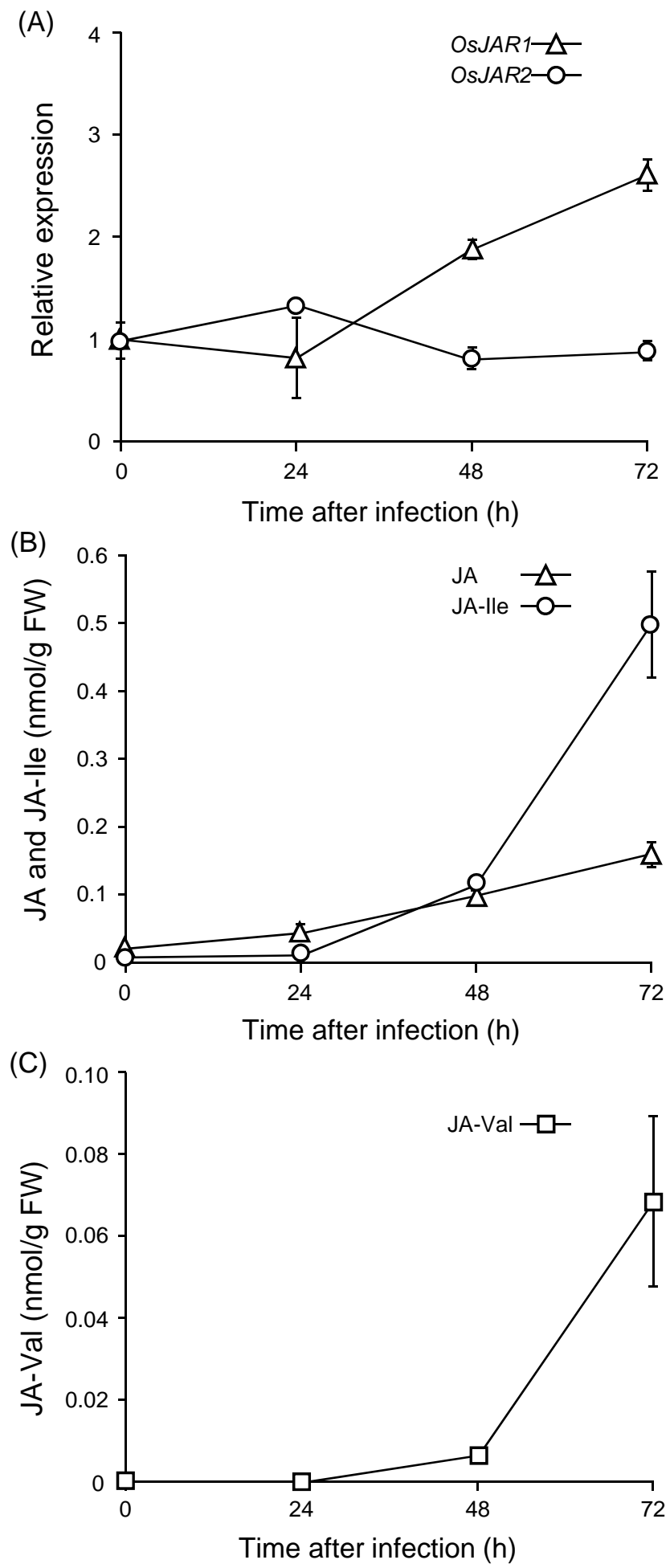

Fig. 4. 
1 : - - - - - MTICSCEETTNEFEMLTRDAARVQKDTLKKILEINASAEYLQNFGLGG- - RTDAESYKSCIPLCVHNDI OSJAR2 1 : MLEKKATRSTRVDGVSGEAVIEEFERVTRDAANVQRETLRRILAENGGVEYLRGLGLAG - - ATDPATFRARVPLATHADL AT JAR1 1 : $\quad-\ldots$ - - MLEKVETFDMNRVIDEFDEMTRNAHQVQKQTLKEILLKNQSAIYLQNCGLNGNATDPEEAFKSMVPLVTDVEL

OsJAR1 68 : EPYTQRIVDGDTSPVVTGEPITNLSLSSGTTHGKPKFIPFNDELLETTLQIYRTSYAFRNREYPIGQ-GKALQFVYGSKQ OSJAR2 79 : EPYIDRIADGDASPVLTAKPATSISLSSGTTQGKRKYLLFNEELVKSTMQIYRISYAFRNREFPVEN-GKALQFIYSSRE AtJAR1 74 : EPYIKRMVDGDTSPILTGHPVPAISLSSGTSQGRPKFIPFTDELMENTLQLFRTAFAFRNRDFPIDDNGKALQFIFSSKQ

OSJAR1 147 : VITKGEILATIATINLYYRRQRY|KEG|VIKDIQSQCCSPDEVIFGGPDFHQSLYCHLLCGLIYSEEVHSVFST|FAHSLVHAFQI OSJAR2 158 : TRTKGGLTATTATTNVYRSEEFKATMRDIQSQCCSPDEVIFGPDFAQSLYCHLLAGLLAAGDVQIVSATFAHSVVLAFQT At JAR1 154 : YISTGGVPVGTATTNVYRNPNFKAGMKSITSPSCSPDEVIFSPDVHOALYCHLLSGILFRDQVQYVFAVFAHGLVHAFRT

OSJAR1 227 : FEEVWEDLCTDIRDG-VLSKKVTAPSIREAVSKILKP-NPELADSIYKKCIGLSNWYGVIPALWPNAKYVYGIMTGSMEP OSJAR2 238 : FERAWEDLCADIRRGEVSPSRVTSPAVRRAMAALLAAPNPGLADEVARKCAALSNWYGVIPALWPNARYVYGIMTGSMEH At JAR1 234 : FEQVWEEIVTDIKDG-VLSNRITVPSVRTAMSKLLTP-NPELAETIRTKCMSLSNWYGLIPALFPNAKYVYGIMTGSMEP OSJAR1 305 : YLKKLRHYAGNLPLISADYYASEGWVGSNIDPTVPPEQVTYAVLPQVGYFEFIPLEKPIGEETENSASIHYIESDPVGLT OSJAR2 318 : YVKKLRHYAGGLPLVAAEYGASEGWVGANVEPGTPPERATFTVLPDIAYFEFIPLKPVAGDGGY - - - - AEAEPVGLT AtJAR1 312 : YVPKLRHYAGDLPLVSHDYGSSEGWIAANVTPRLSPEEATFAVIPNLGYFEFLPVSETGEGEEK - . . - . - - PVGLT

\section{Motif 3}

OSJAR1 385 : EVEVGKIYEVVITNFAGLYRYRLGDVVKIARFHNSTPELQFICRRSLVLSINIDKNTEKDLQLAVEEASKFLEG-EKLEV OSJAR2 391 : EVAAGELYEVVMTTFAGLYRYRLGDVVKVAGFYNATPKLKFVCRRNLMLSINIDKNSEQDLQLAVDAAARAVLAGEKLEV AtJAR1 381 : QVKIGEEYEVVITNYAGLYRYRLGDVVKVIGEYNNTPQLKFICRRNLILSINIDKNTERDLQLSVESAAKRLSE-EKIEV

OSJAR1 464 : MDFTSFVERSSDPGRYVIFWELSG - - - DASDEVLSSCANALDLAFIDAGYTGSRKIKTTGPLELRILRKGTFKEILDHF OSJAR2 471 : VDYTSHADVSSDPGHYVVFLELNAADPAAVDGDVMQACCDELDRAFADAGYVGSRKSGAIAPLELRVLQRGTFQKVLRHY At JAR1 460 : IDFSSYIDVSTDPGHYAIFWEISG - - - ETNEDVLQDCCNCLDRAFIDAGYVSSRKCKTIGALELRVVAKGTFRKIQEHF

OSJAR1 540 : [LSLGGAVSQFKTPRFVNPSNSKVLQILSRNVTQSYFSTAYGF OSJAR2 551 : LSLGAPVSQFKSPRCVSRSNSGVLQILAGCTVNVFFSSAYDAT JAR1 536 : LGLGSSAGOFKMPRCVKPSNAKVLOILCENVVSSYFSTAF - - 
Supplementary Table 1. Primer sequences used for the cloning of OsJAR1, OsJAR2.1, OsJAR2.2, and OsJAR3

\begin{tabular}{ll} 
Primer name & Primer sequence \\
\hline OsJAR1-F & 5'-GCCAGGATTCTACCATTACAATGACG-3' \\
OsJAR1-R & 5'-TGTATCAAAATCCATAGGCAGTACTG-3' \\
OsJAR2.1-F & 5'-ATGTTGGTCGATCGTGCAGG-3' \\
OsJAR2.1-R & 5'-GCGTCTTTGGTTTGCAATTCG-3' \\
OsJAR2.2-F & 5'-GGCGAGATGTTGGAGAAGAAGGC-3' \\
OsJAR2.2-R & 5'-TTATTTTTGGGCAAACATTATATGGCC-3' \\
OsJAR3-F & 5'-GATCCTCTTGCACATTGTTGCAG-3' \\
OsJAR3-R & 5'-CACCACATTCAAACACCGGC-3'
\end{tabular}


Supplementary Table 2. Primer sequences used for the real-time PCR analysis

\begin{tabular}{ll} 
Gene name & Primer sequence \\
\hline OsJAR1 & 5'-AAGGTTTGTGAACCCATCAAACAGC-3' \\
& 5'-AATAATACTTTGCAGCACTTGTTACG-3' \\
OsJAR2 & 5'- CGCCACTACCTCTCCCTCGGCG- \\
& 3' \\
& 5'-GCGTCTTTGGTTTGCAATTCG-3' \\
18S rRNA & 5'-CTACGTCCCTGCCCTTTGTACA-3' \\
& 5'-ACACTTCACCGGACCATTCAA-3'
\end{tabular}

a [41] 
Supplemental Fig. 1. Multiple alignment of amino acid sequences of OsJAR1, OsJAR2, and AtJAR1.

Amino acid sequences were aligned using the ClustalW program. Database accession numbers are as follows: OsJAR1, LOC_Os05g50890.1; OsJAR2, LOC_Os01g12160.1; and AtJAR1, NP_850453. Conserved residues are highlighted in black. The bars above the sequences show the three conserved motifs responsible for ATP/AMP binding. 